\title{
大维乘积随机矩阵谱分布在因子分析 中的应用
}

\author{
金百锁 $1^{*}$ 缪柏其 ${ }^{1}$ 叶五一 ${ }^{1}$ 吴振翔 2
}

(1. 中国科学技术大学统计与金融系, 合肥 $230026 ; 2$. 中国科学院数学与系统科学研究院, 北京 100080)

摘要建立了一个简单实用的估计大维因子模型的因子个数方法. 特别当残差服从线性 时间序列模型时, 给出了其参数的估计方法. 另外也给出了这些估计量的极限理论性质. 最后 的模拟结果和实证分析显示本方法是有意义和有效的.

关键词 大维因子模型 大维乘积随机矩阵 谱分布 因子个数估计 时间序列 $\operatorname{MSC}(2000)$ 主题分类 $60 \mathrm{~F} 15,62 \mathrm{H} 99$

\section{1 引言}

令 $F, \Lambda, r$ 和 $\mathbf{e}$ 定义真实的共同因子、因子负荷、因子个数和残差阵. 因子模型为

$$
\begin{gathered}
X \\
N \times T
\end{gathered} \begin{array}{cc}
\Lambda^{\prime} & F \\
N \times r & r \times T
\end{array} \quad \underset{N \times T}{\mathbf{e},}
$$

在这里共同因子是随机矩阵, 因子负荷为常数矩阵. 在这篇文章里我们讨论大维的情形, 即 $N \rightarrow \infty, T / N \rightarrow y \in(0, \infty)$. 例如在金融中就可以理解为随着时间 $T$ 的增加, 资产种类 $N$ 也 在增加. 在实际中当 $N, T>100$ 一般就可以看成是大维的情形.

在经典的因子模型中矩阵 $\mathbf{e}$ 中的元素相互独立, 矩阵 $F$ 中元素相互独立, 且矩阵 $\mathbf{e}$ 和 $F$ 独立, 隐含的一个条件就是矩阵 $X$ 中的 $T$ 个列向量相互独立, 但在处理一些实际情形时, 这 个条件并不满足. 例如在 $N \times T$ 维金融数据 $X$ 中, $T$ 为时间, $N$ 为资产种类, 我们知道不同 时间金融数据是不独立的, 因此本文讨论的都是 $F$ 中元素相互独立而随机矩阵 $\mathbf{e}$ 中的列向 量不相互独立的情形.

大维因子模型研究并不多, 对于因子个数的估计: 最近在文献 [1] 中采用信息准则方法. 在文献 [2] 中应用了大维样本协方差矩阵最大特征根的方法. 而 Onatski ${ }^{[3]}$ 用特征根的经验 谱分布方法估计因子个数. 本文采用大维样本协方差矩阵特征根的性质来估计因子个数. 与 文献 $[1,2]$ 比较, 文献 [3] 结果更加简单、有效, 因此我们最后只需与 Onatski ${ }^{[3]}$ 的结果相比较, 这里采用与 Onatski 相同的数据, 发现我们的方法能达到同样的效果, 个别还要好一些. 而我 们的方法只需一个待定参数, Onatski 需要两个. 从模拟结果可以看出, 这些参数的选取各有 优缺点. 
本文第 2 个重要的贡献就是讨论矩阵 $\mathrm{e}$ 中列向量之间服从一个线性时间序列时的参数 估计问题, 本文是第 1 篇讨论这个参数估计问题的文章. 以金融数据 $X$ 为例, 在模型 (1) 中, 如果 $\mathbf{e}$ 中列向量之间服从一个线性时间序列, $F$ 中元素相互独立, 则可以理解为把金融数据 $X$ 分为两个部分, 一个是与时间无关部分 (因子负荷乘以共同因子), 另一个与时间相关部分 $\mathrm{e}$, 因此我们对于时间相关部分 $\mathrm{e}$ 的研究就有相当重要的意义.

$\mathrm{Bai}^{[4]}$ 证明了在一些确定性的条件下 $F$ 和 $\Lambda$ 可以用主成分方法得到相合估计. 本文不 讨论 $F$ 和 $\Lambda$ 的估计, 因为不论是因子个数估计还是上面的参数估计方法, 并不需要先得到共 同因子和因子负荷的估计, 相反我们得出的结论会对共同因子和因子负荷的精确估计提供帮 助.

\section{2 因子个数估计}

为了后面证明的需要, 首先给出随机矩阵谱分布的定义. 对于一个 $n \times n$ 维随机 Hermit 矩阵 $A$ 的谱分布定义为

$$
F^{A}(x)=\frac{1}{n} \sum_{j=1}^{n} I\left(\lambda_{j} \leqslant x\right),
$$

其中 $\lambda_{1}, \ldots, \lambda_{n}$ 为随机矩阵 $A$ 的特征根, $I\left(\lambda_{j} \leqslant x\right)$ 为示性函数, 即当 $\lambda_{j} \leqslant x$ 时 $I\left(\lambda_{j} \leqslant x\right)$ 取 值为 1 , 否则为 0 .

在模型 (1) 中我们可以有如下假设:

假设 1. $X$ 中元素 $x_{i, t}(i=1, \ldots, N, t=1, \ldots, T)$ 均值为零, $T / N \rightarrow y \in(0, \infty)$.

如果不为零我们可以考虑差分后的数据. 例如在金融数据中一般考虑回报率, 即如果 $Y_{t}$ 为收益, 回报率为

$$
X_{t}=\log \left(Y_{t}\right)-\log \left(Y_{t-1}\right) \approx \frac{Y_{t}-Y_{t-1}}{Y_{t-1}},
$$

而通常假定回报率均值为零.

假设 $2.0<r<r_{\max }, r_{\max } / T \rightarrow 0, T \rightarrow \infty$.

假设 3. $F$ 中元素 $f_{k, t}(k=1, \ldots, r, t=1, \ldots, T)$ 相互独立, 均值为 0 方差为 1 . 我们知道 在因子分析中因子负荷和共同因子并不唯一. 在这里讨论这样的因子负荷和共同因子, 即如果 $\tilde{L}$ 和 $\tilde{F}$ 是一对真实的因子负荷和共同因子, 乘以一个正交阵 $Q_{r \times r}$, 使得 $Q_{r \times r} * \tilde{L} * \tilde{L}^{\prime} * Q_{r \times r}^{\prime}=$ $\operatorname{diag}\left\{\lambda_{1}, \ldots, \lambda_{r}\right\}$, 则取 $\Lambda=Q_{r \times r} * \tilde{L}$ 和 $F=Q_{r \times r} \tilde{F}$ 为所讨论的因子负荷与共同因子.

假设 4 . 当 $N \rightarrow \infty, \Lambda \Lambda^{\prime}$ 的最小特征根趋于无穷, 即 $\lambda_{\min }\left(\Lambda \Lambda^{\prime}\right) \rightarrow \infty$.

如果令 $\Lambda=\left(\lambda_{k, i}\right)_{r \times N}$, 由假设 3 ,

$$
\lambda_{\min }\left(\Lambda \Lambda^{\prime}\right)=\min \left\{\sum_{i=1}^{N} \lambda_{k, i}^{2}=\lambda_{k}, k=1, \ldots, r\right\} .
$$

以金融数据为例, 第 $k$ 个共同因子对第 $i$ 个资产的影响大小一般用 $\lambda_{k, i}^{2}$ 的大小来表示, 而 $\sum_{i=1}^{N} \lambda_{k, i}^{2}=\lambda_{k}$ 一般称之为第 $k$ 个共同因子对这 $N$ 个资产的累积贡献. 所以这个条件的含义 就是随着资产数量 $N$ 的增加, 任何一个共同因子对资产的累积贡献只会增加, 且增加量不会 衰弱, 即上面级数不收玫.

假设 5. $\mathbf{e}=\Sigma W$, 这里 $\Sigma=\left(\varepsilon_{i, t}\right)_{N \times T}, \varepsilon_{i, t}(i=1, \ldots, N, t=1, \ldots, T)$ 相互独立, 均值为 0 方差为 1 , 四阶矩有限, 且与 $f_{k, t}(k=1, \ldots, r, t=1, \ldots, T)$ 相互独立. $W$ 为 $T \times T$ 维随机 
矩阵与 $\Sigma$ 独立, 且 $\frac{1}{T} W^{\prime} \Sigma^{\prime} \Sigma W$ 的谱分布 (定义 (2)) 收玫到一个非随机分布, 其密度函数单峰 连续, 其最大特征根与最小特征根以概率 1 有界. 例如文献 $[5,6]$ 的多元 $\mathrm{F}$ 矩阵的极限谱分布 和文献 [7] 定理 1.2 的极限谱分布密度函数满足这样的条件. 这里可以看出对任意固定的 $i$, $e_{i, t}(t=1, \ldots, T)$ 不独立.

定理 1 在假设 1-5 成立的条件下, 如果令

$\hat{r}=\max _{i}\left\{i: \lambda_{i}\left(X^{\prime} X / N\right)-\lambda_{i+1}\left(X^{\prime} X / N\right)>\lambda_{i+1}\left(X^{\prime} X / N\right)-\lambda_{i+r_{\alpha}}\left(X^{\prime} X / N\right), i=1, \ldots, r_{\max },\right\}$,

其中 $\lambda_{i}\left(X^{\prime} X / N\right)$ 为矩阵 $X^{\prime} X / N$ 的第 $i$ 大特征根, $r_{\alpha}$ 为不超过 $\alpha T$ 的最大整数, $\alpha$ 为任意小 的一个正常数, 则有 $\hat{r} \stackrel{\text { a.s. }}{\longrightarrow} r$.

证明 由假设 5 , 以概率 $1, \lambda_{1}\left(e^{\prime} e / N\right)<\infty$. 由假设 $2, F F^{\prime} / T \stackrel{\text { a.s. }}{\longrightarrow} I_{r}$, 则

$$
\lambda_{r}\left(F^{\prime} \Lambda \Lambda^{\prime} F / N\right) \stackrel{\text { a.s. }}{\longrightarrow} \infty \text {. }
$$

因此, 对 $i=1, \ldots, r$,

$$
\lambda_{i}\left(X^{\prime} X / N\right)^{1 / 2} \geqslant \lambda_{r}\left(X^{\prime} X / N\right)^{1 / 2} \geqslant \lambda_{r}\left(F^{\prime} \Lambda \Lambda^{\prime} F / N\right)^{1 / 2}-\lambda_{1}\left(e^{\prime} e / N\right)^{1 / 2} \stackrel{\text { a.s. }}{\longrightarrow} \infty ;
$$

在假设 5 之下, $F^{e^{\prime} e / N}(x) \longrightarrow G(x)$, 这里 $G(x)$ 是一个分布函数. $F^{e^{\prime} e / N}(x)$ 为 $e^{\prime} e / N$ 的谱分 布. 由文献 [3] 命题 1 的证明, 对大 $N$ 和 $i=1, \ldots, r_{\text {max }}-r$, 有下面的结果

$$
\begin{aligned}
& \lambda_{i}\left(e^{\prime} e / N\right)-\lambda_{i+1}\left(e^{\prime} e / N\right) \stackrel{\text { a.s. }}{\longrightarrow} 0, \\
& \left.F^{e^{\prime} e / N}\left(\lambda_{i+1}\left(e^{\prime} e / N\right)\right)-F^{e^{\prime} e / N}\left(\lambda_{i+r_{\alpha}}\left(e^{\prime} e / N\right)\right)\right) \\
& \quad=\frac{r_{\alpha}-1}{T} \approx G\left(\lambda_{i+1}\left(e^{\prime} e / N\right)\right)-G\left(\lambda_{i+r_{\alpha}}\left(e^{\prime} e / N\right)\right) \approx \alpha .
\end{aligned}
$$

因此 $i=1, \ldots, r_{\max }$, 以概率 1 有

$$
\lambda_{i}\left(e^{\prime} e / N\right)-\lambda_{i+1}\left(e^{\prime} e / N\right)<\lambda_{i+1}\left(e^{\prime} e / N\right)-\lambda_{i+r_{\alpha}}\left(e^{\prime} e / N\right) .
$$

另一方面, 由 $\operatorname{rank}\left(\Lambda^{\prime} F\right)=r$ 和 $i=r+1, \ldots, r_{\alpha}+r_{\max }$, 使用不等式

$$
\lambda_{i}\left(X^{\prime} X / N\right)^{1 / 2} \leqslant \lambda_{i-r}\left(e^{\prime} e / N\right)^{1 / 2}+\lambda_{r+1}\left(F^{\prime} \Lambda \Lambda^{\prime} F / N\right)^{1 / 2}=\lambda_{i-r}\left(e^{\prime} e / N\right)^{1 / 2}<\infty
$$

和

$$
\lambda_{i}\left(X^{\prime} X / N\right)^{1 / 2} \geqslant \lambda_{i+r}\left(e^{\prime} e / N\right)^{1 / 2}-\lambda_{r+1}\left(F^{\prime} \Lambda \Lambda^{\prime} F / N\right)^{1 / 2}=\lambda_{i+r}\left(e^{\prime} e / N\right)^{1 / 2},
$$

由文献 [3] 命题 1 的证明, 在条件 $r / T \rightarrow 0$ 之下,

$$
\lambda_{i+r}\left(e^{\prime} e / N\right)-\lambda_{i-r}\left(e^{\prime} e / N\right) \stackrel{\text { a.s. }}{\longrightarrow} 0,
$$

我们得到

$$
\lambda_{i}\left(X^{\prime} X / N\right)-\lambda_{i}\left(e^{\prime} e / N\right) \stackrel{\text { a.s. }}{\longrightarrow} 0 .
$$

因此以概率 1 , 当 $i=r+1, \ldots, r_{\text {max }}$ 时,

$$
\left(\lambda_{i}\left(X^{\prime} X / N\right)-\lambda_{i+1}\left(X^{\prime} X / N\right)\right)<\left(\lambda_{i+1}\left(X^{\prime} X / N\right)-\lambda_{i+r_{\alpha}}\left(X^{\prime} X / N\right)\right) .
$$

综合 (3) 和 (4) 式

$$
\hat{r} \stackrel{\text { a.s. }}{\longrightarrow} r .
$$

定理证毕. 


\section{3 残差是时间序列时其参数的估计}

在这小节中我们假设残差 $\left\{e_{i, t}, t=1, \ldots, T\right\}$ 为线性时间序列 $\operatorname{ARMA}(p, q)$, 即

$$
e_{i, t+1}=b_{1} e_{i, t}+\cdots+b_{p} e_{i, t+1-p}+a_{1} \varepsilon_{i, t+1}+\cdots+a_{q} \varepsilon_{i, t+2-q},
$$

这里 $a_{1}, \ldots, a_{q}, b_{1}, \ldots, b_{p}$ 为常数, 可以写成矩阵形式.

令

$$
\mathbf{e}_{i}^{\prime}=\left(\begin{array}{c}
e_{i, 1} \\
e_{i, 2} \\
\vdots \\
e_{i, p} \\
e_{i, p+1} \\
\vdots \\
e_{i, T}
\end{array}\right)=b_{1}\left(\begin{array}{c}
0 \\
e_{i, 1} \\
\vdots \\
e_{i, p-1} \\
e_{i, p} \\
\vdots \\
e_{i, T-1}
\end{array}\right)+\cdots+b_{p}\left(\begin{array}{c}
0 \\
0 \\
\vdots \\
0 \\
e_{i, 1} \\
\vdots \\
e_{i, T-p}
\end{array}\right)+a_{1}\left(\begin{array}{c}
\varepsilon_{i, 1} \\
\varepsilon_{i, 2} \\
\vdots \\
\varepsilon_{i, p} \\
\varepsilon_{i, p+1} \\
\vdots \\
\varepsilon_{i, T}
\end{array}\right)+\cdots+a_{q}\left(\begin{array}{c}
0 \\
0 \\
\vdots \\
0 \\
\varepsilon_{i, 1} \\
\vdots \\
\varepsilon_{i, T+1-q}
\end{array}\right),
$$

我们有

$$
\begin{aligned}
& \mathbf{e}_{i}^{\prime}=\left(\begin{array}{cccccc}
0 & 0 & \cdots & \cdots & \cdots & 0 \\
b_{1} & 0 & 0 & \cdots & \cdots & 0 \\
\vdots & \ddots & \vdots & \ddots & \ddots & \vdots \\
b_{p} & \ldots & b_{1} & 0 & \ldots & 0 \\
\vdots & \ddots & \vdots & \ddots & \ddots & \vdots \\
0 & \ldots & b_{p} & \ldots & b_{1} & 0
\end{array}\right) \mathbf{e}_{i}^{\prime}+\left(\begin{array}{cccccc}
a_{1} & 0 & \cdots & \cdots & \cdots & 0 \\
a_{2} & a_{1} & 0 & \cdots & \cdots & 0 \\
\vdots & \ddots & \ddots & \ddots & \ddots & \vdots \\
a_{q} & \cdots & a_{2} & a_{1} & \cdots & 0 \\
\vdots & \ddots & \ddots & \ddots & \ddots & \vdots \\
0 & \cdots & a_{q} & \cdots & a_{2} & a_{1}
\end{array}\right)\left(\begin{array}{c}
\varepsilon_{i, 1} \\
\varepsilon_{i, 2} \\
\vdots \\
\varepsilon_{i, q+1} \\
\vdots \\
\varepsilon_{i, T}
\end{array}\right) \\
& =\left(\begin{array}{cccccc}
1 & 0 & \cdots & \ldots & \cdots & 0 \\
-b_{1} & 1 & 0 & \ldots & \ldots & 0 \\
\vdots & \ddots & \vdots & \ddots & \ddots & \vdots \\
-b_{p} & \cdots & -b_{1} & 1 & \ldots & 0 \\
\vdots & \ddots & \vdots & \ddots & \ddots & \vdots \\
0 & \cdots & -b_{p} & \cdots & -b_{1} & 1
\end{array}\right)\left(\begin{array}{cccccc}
a_{1} & 0 & \ldots & \ldots & \cdots & 0 \\
a_{2} & a_{1} & 0 & \cdots & \cdots & 0 \\
\vdots & \ddots & \vdots & \ddots & \ddots & \vdots \\
a_{q} & \cdots & a_{2} & a_{1} & \ldots & 0 \\
\vdots & \ddots & \vdots & \ddots & \ddots & \vdots \\
0 & \ldots & a_{q} & \ldots & a_{2} & a_{1}
\end{array}\right)\left(\begin{array}{c}
\varepsilon_{i, 1} \\
\varepsilon_{i, 2} \\
\vdots \\
\varepsilon_{i, q+1} \\
\vdots \\
\varepsilon_{i, T}
\end{array}\right) \\
& \equiv\left(\begin{array}{cccccc}
c_{0} & 0 & \cdots & \cdots & \cdots & 0 \\
c_{1} & c_{0} & 0 & \cdots & \cdots & 0 \\
\vdots & \ddots & \vdots & \ddots & \ddots & \vdots \\
c_{p} & \cdots & c_{1} & c_{0} & \cdots & 0 \\
\vdots & \ddots & \vdots & \ddots & \ddots & \vdots \\
c_{T-1} & \cdots & c_{p} & \cdots & c_{1} & c_{0}
\end{array}\right)\left(\begin{array}{c}
\varepsilon_{i, 1} \\
\varepsilon_{i, 2} \\
\vdots \\
\varepsilon_{i, p+1} \\
\vdots \\
\varepsilon_{i, T}
\end{array}\right) \equiv \mathbf{C} \Sigma_{i}^{\prime}
\end{aligned}
$$

因此 $\mathbf{e}=\Sigma \mathbf{C}^{\prime}$. 可以看出这个结果是假设 5 的一个特例, 即随机矩阵 $W$ 用常数矩阵 $\mathbf{C}$ 代替.

例如当 $p=q=1$, 可以得到

$$
c_{0}=a_{1}, \quad c_{k}=b_{1}^{k-1} z, \quad z=a_{1} b_{1}+a_{2}, \quad i=1, \ldots, T, \quad k=1, \ldots, T-1 .
$$


因此我们可以把上面的因子模型写成如下的矩阵形式:

$$
\begin{aligned}
\left(\begin{array}{ccc}
x_{1,1} & \cdots & x_{1, T} \\
\vdots & \ddots & \vdots \\
x_{N, 1} & \cdots & x_{N, T}
\end{array}\right)= & \left(\begin{array}{ccc}
\lambda_{1,1} & \ldots & \lambda_{1, r} \\
\vdots & \ddots & \vdots \\
\lambda_{N, 1} & \cdots & \lambda_{N, r}
\end{array}\right)\left(\begin{array}{ccc}
f_{1,1} & \ldots & f_{1, T} \\
\vdots & \ddots & \vdots \\
f_{r, 1} & \ldots & f_{r, T}
\end{array}\right) \\
& +\left(\begin{array}{ccc}
\varepsilon_{1,1} & \cdots & \varepsilon_{1, T} \\
\vdots & \ddots & \vdots \\
\varepsilon_{N, 1} & \cdots & \varepsilon_{N, T}
\end{array}\right)\left(\begin{array}{ccc}
c_{0} & \cdots & c_{T-1} \\
\vdots & \ddots & \vdots \\
0 & \cdots & c_{0}
\end{array}\right)
\end{aligned}
$$

这个结果就是把观测样本 $\mathbf{X}$ 分隔成行相关 (独立随机阵左乘一个常数阵) 和列相关 (独 立随机矩阵右乘一个上三角阵, 因为只能是前面时刻的影响后面的反之不成立) 两个部分. 观 察模型 (5), 我们看到这里讨论的 $b_{1}, \ldots, b_{p}, a_{1}, \ldots, a_{q}$ 与 $i$ 无关, 如果为金融数据, 即其反映 的是由 $N$ 个资产组成的整个市场的共同时间波动性质, 如果波动大说明这个由 $N$ 个资产组 成的整个市场活跃, 如果波动小说明不活跃.

综合上面我们有以下假设:

假设 6 . 对每个固定的 $i,\left\{e_{i, t}\right\}$ 满足模型 (5) 且是因果可逆的. 模型 (5) 中 $\varepsilon_{i t}$ 相互独立, 均值为 0 方差为 1 , 有有限的四阶矩, 且与 $f_{i t}$ 独立.

假设 7 . 等式 (7) 中, $\sum_{i=0}^{T-1} c_{i}^{2}<\infty$.

例如当 $p=q=1$, 在假设 6 下 $\left|b_{1}\right|<1, c_{0}=a_{1}, c_{k}=b_{1}^{k-1} z, z=a_{1} b_{1}+a_{2}, i=1, \ldots, T, k=$ $1, \ldots, T-1$,

$$
\sum_{i=0}^{T-1} c_{i}^{2}=a_{1}^{2}+\sum_{k=1}^{T-1} b_{1}^{2 k-2} z^{2} \rightarrow a_{1}^{2}+\frac{z^{2}}{1-b_{1}^{2}}<\infty .
$$

引理 1 如果假设 6 成立, 在等式 (7) 中 $p=q=1$, 则有下面 3 个结论:

$$
\begin{aligned}
& \operatorname{tr} \mathbf{C}^{\prime} \mathbf{C} / T \rightarrow a_{1}^{2}+\frac{\left(a_{1} b_{1}+a_{2}\right)^{2}}{1-b_{1}^{2}}, \\
& \operatorname{tr}\left(\mathbf{C}^{\prime} \mathbf{C}\right)^{2} / T \rightarrow \frac{\left(a_{1}^{2}+a_{2}^{2}+2 a_{1} b_{1} a_{2}\right)^{2}}{\left(b_{1}^{2}-1\right)^{2}}+2 \frac{\left(a_{1}+b_{1} a_{2}\right)^{2}\left(a_{1} b_{1}+a_{2}\right)^{2}}{\left(1-b_{1}^{2}\right)^{3}},
\end{aligned}
$$

和

$$
\begin{aligned}
\operatorname{tr}\left(\mathbf{C}^{\prime} \mathbf{C}\right)^{3} / T \longrightarrow \frac{\left(a_{1}^{2}+a_{2}^{2}+2 a_{1} b_{1} a_{2}\right)^{3}}{\left(1-b_{1}^{2}\right)^{3}} & \\
& +\frac{6\left(a_{1}+b_{1} a_{2}\right)^{2}\left(a_{1} b_{1}+a_{2}\right)^{2}\left(a_{1}^{2}+a_{2}^{2}+2 a_{1} b_{1} a_{2}\right)}{\left(1-b_{1}^{2}\right)^{4}}+\frac{6\left(a_{1}+b_{1} a_{2}\right)^{3}\left(a_{1} b_{1}+a_{2}\right)^{3} b 1}{\left(1-b_{1}^{2}\right)^{5}} .
\end{aligned}
$$

证明从 (7) 式, 如果记 $\mathbf{C}=\left(\sigma_{i, j}\right)_{T \times T}$, 则得到

$$
\begin{gathered}
\sigma_{i, i}=c_{0}=a_{1}, \quad \sigma_{i+k, i}=c_{k}=b_{1}^{k-1} z, \quad z=a_{1} b_{1}+a_{2}, \\
i=1, \ldots, T, \quad k=1, \ldots, T-i, \quad \sigma_{i, j}=0, \quad i<j .
\end{gathered}
$$

令 $\mathbf{C}^{\prime} \mathbf{C}=\left(S_{i, j}\right)_{T \times T}$, 则

$$
S_{i, i}=\sum_{k=1}^{i} \sigma_{i, k}^{2}=a_{1}^{2}+\sum_{k=1}^{i-1} b_{1}^{2(i-k)-2} z^{2},
$$


因此当 $i \rightarrow \infty$ 时, $S_{i, i} \rightarrow a_{1}^{2}+z^{2} /\left(1-b_{1}^{2}\right)$. 同理当 $i \neq j$ 时,

$$
S_{i, j}=\sum_{k=1}^{\min \{i, j\}} \sigma_{i, k} \sigma_{j, k}=\left(a_{1} z+\sum_{k=1}^{\min \{i, j\}-1} z^{2} b_{1}^{i+j-|i-j|-2 k-1}\right) b_{1}^{|i-j|-1} .
$$

由于 $\operatorname{tr} \mathbf{C}^{\prime} * \mathbf{C} / T=\sum_{i=1}^{T} S_{i, i} / T$, 因此由简单的数学分析知识, 有

$$
\operatorname{tr}\left(\mathbf{C}^{\prime} \mathbf{C}\right) / T \rightarrow a_{1}^{2}+\frac{\left(a_{1} b_{1}+a_{2}\right)^{2}}{1-b_{1}^{2}} .
$$

第 1 个结论证毕.

又

$$
\operatorname{tr}\left(\mathbf{C}^{\prime} \mathbf{C}\right)^{2} / T=\sum_{i=1}^{T} \sum_{j=1}^{T} S_{i, j}^{2} / T=\sum_{i=1}^{T} S_{i, i}^{2} / T+2 \sum_{i=1}^{T} \sum_{j=1}^{i-1} S_{i, j}^{2} / T
$$

而

$$
\sum_{i=1}^{T} S_{i, i}^{2} / T \rightarrow\left(a_{1}^{2}+\frac{\left(a_{1} b_{1}+a_{2}\right)^{2}}{1-b_{1}^{2}}\right)^{2}
$$

因为当 $\min \{i, j\} \rightarrow \infty$ 时,

$$
a_{1} z+\sum_{k=1}^{\min \{i, j\}-1} z^{2} b_{1}^{i+j-|i-j|-2 k-1} \rightarrow a_{1} z+z^{2} b_{1} /\left(1-b_{1}^{2}\right),
$$

所以当 $T \rightarrow \infty$ 时,

$$
\sum_{i=1}^{T} \sum_{j=1}^{i-1} S_{i, j}^{2} / T=\sum_{i=1}^{T} \sum_{j=1}^{i-1}\left(a_{1} z+\sum_{k=1}^{\min \{i, j\}-1} z^{2} b_{1}^{i+j-|i-j|-2 k-1}\right)^{2} b_{1}^{2|i-j|-2} / T
$$

与

$$
\left(a_{1} z+z^{2} b_{1} /\left(1-b_{1}^{2}\right)\right)^{2} \sum_{i=1}^{T} \sum_{j=1}^{i-1} b_{1}^{2|i-j|-2} / T
$$

有相同极限分布.

进而可以证明

$$
\sum_{i=1}^{T} \sum_{j=1}^{i-1} b_{1}^{2|i-j|-2} / T=\sum_{i=1}^{T-1}(T-i) b_{1}^{2 i-2} / T \rightarrow \frac{1}{1-b_{1}^{2}},
$$

因此

$$
\sum_{i=1}^{T} \sum_{j=1}^{i-1} S_{i, j}^{2} / T \rightarrow \frac{\left(a_{1}+b_{1} a_{2}\right)^{2}\left(a_{1} b_{1}+a_{2}\right)^{2}}{\left(1-b_{1}^{2}\right)^{3}}
$$

第 2 个结论证毕.

最后

$$
\begin{aligned}
\operatorname{tr}\left(\mathbf{C}^{\prime} \mathbf{C}\right)^{3} / T & =\sum_{i=1}^{T} \sum_{j=1}^{T} \sum_{k=1}^{T} S_{i, j} S_{j, k} S_{k, i} / T \\
& =\sum_{i=1}^{T} S_{i, i}^{3} / T+6 \sum_{i=1}^{T} \sum_{j=1}^{i-1} S_{i, i} S_{i, j}^{2} / T+6 \sum_{T \geqslant i>j>k \geqslant 1} S_{i, j} S_{j, k} S_{k, i} / T .
\end{aligned}
$$


我们知道

$$
\sum_{i=1}^{T} S_{i, i}^{3} / T \rightarrow\left(a_{1}^{2}+\frac{\left(a_{1} b_{1}+a_{2}\right)^{2}}{1-b_{1}^{2}}\right)^{3}
$$

当 $T \rightarrow \infty$ 时,

$$
\begin{aligned}
& \sum_{i=1}^{T} \sum_{j=1}^{i-1} S_{i, i} S_{i, j}^{2} / T \\
& \left(a_{1}^{2}+\frac{\left(a_{1} b_{1}+a_{2}\right)^{2}}{1-b_{1}^{2}}\right) \sum_{i=1}^{T} \sum_{j=1}^{i-1} S_{i, j}^{2} / T
\end{aligned}
$$

有相同极限, 因此由 (8) 式,

$$
\sum_{i=1}^{T} \sum_{j=1}^{i-1} S_{i, i} S_{i, j}^{2} / T \rightarrow \frac{\left(a_{1}+b_{1} a_{2}\right)^{2}\left(a_{1} b_{1}+a_{2}\right)^{2}\left(a_{1}^{2}+a_{2}^{2}+2 a_{1} b_{1} a_{2}\right)}{\left(1-b_{1}^{2}\right)^{4}} .
$$

由 (8) 式的证明知道

$$
\sum_{T \geqslant i>j>k \geqslant 1} S_{i, j} S_{j, k} S_{k, i} / T
$$

与

$$
\left(a_{1} z+\frac{z^{2} b_{1}}{1-b_{1}^{2}}\right)^{3} \sum_{T \geqslant i>j>k \geqslant 1} b_{1}^{2(i-k)-3} / T
$$

有相同的极限. 而

$$
\sum_{T \geqslant i>j>k \geqslant 1} b_{1}^{2(i-k)-3} / T=\sum_{i=2}^{T-1}(i-1)(T-i) b_{1}^{2 i-3} / T \rightarrow \frac{b_{1}}{\left(1-b_{1}^{2}\right)^{2}} .
$$

第 3 个结论证毕.

在 $\operatorname{ARMA}(p, q)$ 模型中, $\operatorname{ARMA}(1,1)$ 模型有一定的代表性, 接下来以 $\operatorname{ARMA}(1,1)$ 为例 讨论其参数的估计, 给出如何用矩估计方法来估计这些参数.

假设 8. 对每个 $i, e_{i, t} \sim \operatorname{ARMA}(1,1)$, 即 $e_{i, t+1}=b_{1} e_{i, t}+a_{1} \varepsilon_{i, t+1}+a_{2} \varepsilon_{i, t}$. 且 $a_{1}, a_{2}, b_{1}$ 都 为非负数. 对于金融数据, 这里假定后面时刻的回报率与前一时刻的正相关, 正如文献 [8] 发 现的回报率具有短期惯性, 这个假定也是为了保证我们下面估计的唯一性. 对具体数据分析 可以先计算不同时刻数据的相关性, 再判断参数的正负.

定义: 令 $\lambda_{1} \geqslant \lambda_{2} \geqslant \cdots \geqslant \lambda_{T}$ 是 $X^{\prime} X / N$ 特征根, $g_{i}=\frac{\sum_{j=r+1}^{T} \lambda_{j}^{i}}{T-r}, i=1,2,3, y=T / N$.

定理 2 如果假设 1-4 和 6-8 满足, 令

$$
\begin{aligned}
& \hat{b}_{1}=\sqrt{2\left(H_{3}+2 H_{1}^{3}-3 H_{2} H_{1}\right)^{2} /\left(2 H_{3}^{2}+9 H_{2}^{3}-H_{1}^{6}+8 H_{3} H_{1}^{3}+3 H_{2} H_{1}^{4}-9 H_{2}^{2} H_{1}^{2}-12 H_{1} H_{2} H_{3}\right)}, \\
& \hat{a}_{1}=\frac{\sqrt{2 f_{1}+f_{2}}+\sqrt{f_{2}-2 f_{1}}}{2}, \hat{a}_{2}=\frac{\sqrt{2 f_{1}+f_{2}}-\sqrt{f_{2}-2 f_{1}}}{2} .
\end{aligned}
$$

这里

$$
\begin{aligned}
& f_{1}=\sqrt{\left(1-\hat{b}_{1}^{2}\right)\left(H_{2}-H_{1}^{2}\right) / 2}-\hat{b}_{1} H_{1}, \quad f_{2}=\left(1+\hat{b}_{1}^{2}\right) H_{1}-\hat{b}_{1} \sqrt{2\left(1-\hat{b}_{1}^{2}\right)\left(H_{2}-H_{1}^{2}\right)}, \\
& H_{1}=g_{1}, \quad H_{2}=g_{2}-y H_{1}^{2}, \quad H_{3}=g_{3}-y^{2} H_{1}^{3}-3 y H_{2} H_{1},
\end{aligned}
$$


则有 $\hat{b}_{1}, \hat{a}_{1}, \hat{a}_{2}$ 为 $b_{1}, a_{1}, a_{2}$ 的强相合估计.

证明 由文献 [9] 定理 2.1 或文献 [7] 定理 1.1 的证明,有

$$
\frac{1}{N} \operatorname{tr}\left[\left(\mathbf{C} \Sigma^{\prime} \Sigma \mathbf{C}^{\prime}\right)\right]^{k} \stackrel{\text { a.s. }}{\longrightarrow} \sum_{s=1}^{k} c^{k-s} \sum_{\substack{i_{1}+\cdots+i_{s}=k-s+1 \\ i_{1}+\cdots+s i_{s}=k}} \frac{k !}{s !} \prod_{m=1}^{s} \frac{D_{m}^{i_{m}}}{i_{m} !},
$$

这里

$$
D_{k}=\lim _{T \rightarrow \infty} \frac{1}{T} \operatorname{tr}\left[\left(\mathbf{C}^{\prime} \mathbf{C}\right)^{k}\right] .
$$

由文献 [7] 定理 1.1 的证明过程可以得到, 如果假设 6 和 7 满足, 则假设 5 成立, 因此由 定理 1 的证明, 有 $g_{i}-\frac{1}{N} \operatorname{tr}\left[\left(\mathbf{C} \Sigma^{\prime} \Sigma \mathbf{C}^{\prime}\right)^{i}\right] \stackrel{\text { a.s. }}{\longrightarrow} 0, i=1,2,3$. 因此由 (9) 式和 $H_{1}=g_{1}, H_{2}=$ $g_{2}-y H_{1}^{2}, H_{3}=g_{3}-y^{2} H_{1}^{3}-3 y H_{2} H_{1}$, 得 $H_{i} \stackrel{\text { a.s. }}{\longrightarrow} D_{i}, i=1,2,3$.

使用引理 1 , 建立方程组

$$
\left\{\begin{aligned}
H_{1}= & \hat{a}_{1}^{2}+\frac{\left(\hat{a}_{1} \hat{b}_{1}+\hat{a}_{2}\right)^{2}}{1-\hat{b}_{1}^{2}}, \\
H_{2}= & \frac{\left(\hat{a}_{1}^{2}+\hat{a}_{2}^{2}+2 \hat{a}_{1} \hat{b}_{1} \hat{a}_{2}\right)^{2}}{\left(\hat{b}_{1}^{2}-1\right)^{2}}+2 \frac{\left(\hat{a}_{1}+\hat{b}_{1} \hat{a}_{2}\right)^{2}\left(\hat{a}_{1} \hat{b}_{1}+\hat{a}_{2}\right)^{2}}{\left(1-\hat{b}_{1}^{2}\right)^{3}}, \\
H_{3}= & \frac{\left(\hat{a}_{1}^{2}+\hat{a}_{2}^{2}+2 \hat{a}_{1} \hat{b}_{1} \hat{a}_{2}\right)^{3}}{\left(1-\hat{b}_{1}^{2}\right)^{3}}+\frac{6\left(\hat{a}_{1}+\hat{b}_{1} \hat{a}_{2}\right)^{2}\left(\hat{a}_{1} \hat{b}_{1}+\hat{a}_{2}\right)^{2}\left(\hat{a}_{1}^{2}+\hat{a}_{2}^{2}+2 \hat{a}_{1} \hat{b}_{1} \hat{a}_{2}\right)}{\left(1-\hat{b}_{1}^{2}\right)^{4}} \\
& +\frac{6\left(\hat{a}_{1}+\hat{b}_{1} \hat{a}_{2}\right)^{3}\left(\hat{a}_{1} \hat{b}_{1}+\hat{a}_{2}\right)^{3} \hat{b}_{1}}{\left(1-\hat{b}_{1}^{2}\right)^{5}} .
\end{aligned}\right.
$$

由假设 6 和 8 , 只需注意 $0 \leqslant \hat{b}_{1}<1,0 \leqslant \hat{a}_{2}<\hat{a}_{1}$ 即可得到唯一解, 进而得出我们的结论. 定 理证毕.

同理对 $\mathrm{AR}(1)$ 和 $\mathrm{MA}(1)$ 这两个模型, 因为只有两个参数, 就可以只用一阶矩和二阶矩来 估计. 结果如下:

推论 1 在定理 2 的条件下, 当 $e_{i, t+1}=b e_{i, t}+a \varepsilon_{i, t+1}$ 时, 有

$$
\begin{aligned}
& \tilde{b}=\sqrt{\left(g_{2}-(T / N+1) g_{1}^{2}\right) /\left(g_{2}+(1-T / N) g_{1}^{2}\right)} \stackrel{\text { a.s. }}{\longrightarrow} b, \\
& \tilde{a}=\sqrt{2 g_{1}^{3} /\left(g_{2}+(1-T / N) g_{1}^{2}\right)} \stackrel{\text { a.s. }}{\longrightarrow} a ;
\end{aligned}
$$

当 $e_{i, t+1}=a_{1} \varepsilon_{i, t+1}+a_{2} \varepsilon_{i, t}$ 时, 有

$$
\begin{aligned}
& \hat{a}_{1}=\sqrt{\frac{g_{1}+\sqrt{(3+2 T / N) g_{1}-2 g_{2}^{2}}}{2}} \stackrel{\text { a.s. }}{\longrightarrow} a_{1}, \\
& \hat{a}_{2}=\sqrt{\frac{g_{1}-\sqrt{(3+2 T / N) g_{1}-2 g_{2}^{2}}}{2}} \stackrel{\text { a.s. }}{\longrightarrow} a_{2} .
\end{aligned}
$$

备注 1 对于实际问题可以先用定理 1 得出因子个数 $r$ 的估计, 再用定理 2 给出 $b_{1}, a_{1}, a_{2}$ 的估计. 在这节只给出了误差序列是 $\operatorname{ARMA}(1,1)$, 其实用我们的方法可以给出其 他一些 $\operatorname{ARMA}(p, q)$ 模型的估计, 这里主要的要找出 $\operatorname{tr}\left(\mathbf{C}^{\prime} \mathbf{C}\right)^{k} / T$ 的极限, 我们的这个结果给 出处理这样问题的一个思路, 希望能有抛砖引玉的作用. 


\section{Monte Carlo 模拟}

在这节, 我们用 Monte Carlo 进行模拟并与文献 [3] 的模拟结果比较. 令 $\lambda_{i}$ 是随机矩阵 $\frac{1}{T} X X^{\prime}$ 的第 $i$ 大特征根. 文献 [3] 定义一些 $r$ 的估计量

$$
\hat{r}_{\delta}=\sharp\left\{i \leqslant n: \lambda_{i}>(1+\delta) \hat{u}\right\},
$$

这里 $\delta$ 是一个正常数,

$$
\hat{u}=w \lambda_{r_{\max }+1}+(1-w) \lambda_{2 r_{\max }+1}, \quad w=2^{2 / 3} /\left(2^{2 / 3}-1\right) .
$$

在这里有两个未知量 $\delta$ 和 $r_{\max }$, 在我们的结果中只要一个未知量 $r_{\alpha}$. 在文献 [3] 中 $\delta$ 取 3 个 值 $0, N^{-1 / 2}, N^{-2 / 5} . r_{\max }$ 根据不同的 $N$ 和 $T$ 取不同的值, 这个值是反复验算得到的. 在这节 我们的模拟模型为

$$
y_{i, t}=\sum_{j=1}^{r_{0}} \lambda_{j, i} f_{j, t}+e_{i, t}, \quad i=1, \ldots, N, \quad t=1, \ldots, T .
$$

$e_{i, t+1}=b_{1} e_{i, t}+a_{1} \varepsilon_{i, t+1}+a_{2} \varepsilon_{i, t}$, 这里

$$
\lambda_{j, i} \sim N(0,1), f_{j, t} \text { iid } \sim N(0,1), \varepsilon_{i, t} \text { iid } \sim N(0,1) .
$$

令 $\lambda_{j, i}$ 固定, $f_{j, t}$ 和 $\varepsilon_{i, t}$ 重复抽样 1000 次, 最后对得到估计取平均.

这里对 $\left\{e_{i, t}\right\} \sim \operatorname{MA}(1),\left\{e_{i, t}\right\} \sim \operatorname{AR}(1),\left\{e_{i, t}\right\} \sim \operatorname{ARMA}(1,1)$ 分别模拟, 对于前两种采 用推论 1 的估计参数 $\hat{a}_{1}, \hat{a}_{2}$ 与 $\hat{a}, \hat{b}$; 对于最后一种采用定理 2 来估计参数 $\hat{a}_{1}, \hat{b}_{1}, \hat{a}_{2}$. 而且 对于每种模型分别应用定理 1 和 (10) 式得到我们对因子个数的估计 $\hat{r}$ 和文献 [3] 的 3 种估 计 $\hat{r}_{0}, \hat{r}_{N^{-1 / 2}}, \hat{r}_{N^{-2 / 5}}$. 而对每个模型我们又分两种, 一种是残差的方差小于共同因子与因 子负荷乘积的方差 (“正常情形”), 即 $\left(a_{1}^{2}+a_{2}^{2}\right) /\left(1-b_{1}^{2}\right) \leqslant r$, 这个条件表明共同因子与因子 负荷乘积是主项, $\left(a_{1}^{2}+a_{2}^{2}\right) /\left(1-b_{1}^{2}\right) \leqslant r$ 可以近似看成满足假设 4 , 模拟结果在附录的表 1 , 表 3 , 表 5 ; 相反另一种是残差的方差大于共同因子与因子负荷乘积的方差 (“异常情形”), 即 $\left(a_{1}^{2}+a_{2}^{2}\right) /\left(1-b_{1}^{2}\right) \gg r$ (在模拟中我们把表 1 , 表 3 , 表 5 中的时间序列参数同时扩大 3 倍, 而 因子个数 $r$ 不变), 即要看看如果完全不满足假设 4 , 我们的结果是否仍然成立, 模拟结果在 附录的表 2 , 表 4 , 表 6 .

另外对于 $N$ 和 $T$ 的选取, 我们采用文献 [3] 的数据, 因为每对数据都有相应的意义, 具体 可参看文献 [3] 的解释. 通过观察下面 6 个表格, 我们惊奇的发现对于因子个数 $r$ 的估计, 我 们的未知量 $r_{\alpha}$, 在 “正常情形” 下的 3 张表格中除了有两个在 $N=40, T=100$ 相对小样本 的情形下 $r_{\alpha}=7$, 其他情形用 $r_{\alpha}=8$, 都可以得到 $r$ 的很精确的估计. 在 “异常情形”下, 我们 $r$ 的估计与文献 [3] 的结果相差不大, 但我们的 $r_{\alpha}$ 的选取就要根据 $N$ 和 $T$ 不同而不同. 然 而文献 [3] 中 $r_{\max }$ 的选取, 是根据 $N$ 和 $T$ 不同而不同的, 与 “正常情形” 或者 “异常情形” 无 关, 因此, 如果共同用文献 [3] 和我们的估计方法, 就能判断是 “正常情形” 还是 “异常情形”, 这一点在下面的实证中用到.

而对于我们时间序列 $\mathrm{AR}(1)$ 和 $\mathrm{MA}(1)$, 不管 “正常情形” 还是 “异常情形”, 其参数估计 都很精确. 而对于 $\operatorname{ARMA}(1,1)$, 其参数估计就不如 $\operatorname{AR}(1)$ 和 $\mathrm{MA}(1)$ 精确, 对条件要求比较 严, 即只有当 $N$ 和 $T$ 都比较大的时候才有很好的估计, 而且不论 “正常情形” 还是 “异常情 形”都无明显影响. 


\section{5 实证分析}

我们对上海和深圳的股票进行分析. 对上海股市, 选取 174 种从 2004 年 3 月 24 日到 2006 年 3 月 24 日两年 485 天的股票数据, 我们计算回报率, 即 $y_{i, t}=\log \left(x_{i, t}\right)-\log \left(x_{i, t-1}\right)$, 其中 $x_{i, t}$ 为第 $i$ 种股票 $t$ 时刻的复权价. 如果残差用 $\operatorname{ARMA}(1,1)$ 模型, 如上面模拟选择 $r_{\text {max }}=12$, $r_{\alpha}=8$. 实证结果如下:

$$
\hat{r}_{0}=8, \quad \hat{r}_{N^{-1 / 2}}=8, \quad \hat{r}_{N^{-2 / 5}}=8, \quad \hat{r}=8, \quad \hat{a}_{1}=0.0215, \quad \hat{b}_{1}=0.961, \quad \hat{a}_{2}=-0.01 .
$$

注意到 $\hat{a}_{2}=-0.01 \approx 0$, 我们用 $\operatorname{AR}(1)$ 模型模拟. 结果如下:

$$
\hat{r}_{0}=8, \quad \hat{r}_{N^{-1 / 2}}=8, \quad \hat{r}_{N^{-2 / 5}}=8, \quad \hat{r}=8, \quad \hat{b}=0.9511, \quad \hat{a}=0.0136 .
$$

即上海股市有 8 个共同因子, 且上海股市的股票去掉外部共同因子影响, 共同波动为 $e_{i, t}=$ $0.9511 e_{i, t-1}+0.0136 \varepsilon_{i, t}$.

对深圳股市, 我们选取 89 种从 2004 年 1 月 2 日到 2006 年 3 月 31 日 541 天的股票数 据, 同样应用回报率, 如果残差用 $\operatorname{ARMA}(1,1)$ 模型, $r_{\max }=12, r_{\alpha}=8$. 实证结果如下:

$$
\hat{r}_{0}=5, \quad \hat{r}_{N^{-1 / 2}}=5, \quad \hat{r}_{N^{-2 / 5}}=4, \quad \hat{r}=1, \quad \hat{a}_{1}=0.0162, \quad \hat{b}_{1}=0.9594, \quad \hat{a}_{2}=-0.0128 .
$$

我们发现我们的 $r$ 的估计与文献 [3] 的 3 种估计相差比较大, 我们选 $r_{\max }=12, r_{\alpha}=4$. 结果 如下:

$$
\hat{r}_{0}=5, \quad \hat{r}_{N^{-1 / 2}}=5, \quad \hat{r}_{N^{-2 / 5}}=4, \quad \hat{r}=5, \quad \hat{a}_{1}=0.0118, \quad \hat{b}_{1}=0.6705, \quad \hat{a}_{2}=0.0014 .
$$

同样注意到 $\hat{a}_{2} \approx 0$, 我们用 $\mathrm{AR}(1)$ 模型模拟, $r_{\max }=12, r_{\alpha}=4$. 结果如下:

$$
\hat{r}_{0}=5, \quad \hat{r}_{N^{-1 / 2}}=5, \quad \hat{r}_{N^{-2 / 5}}=4, \quad \hat{r}=5, \quad \hat{b}=0.7002, \quad \hat{a}=0.0123 .
$$

即深圳股市有 5 个共同因子, 且去掉外部共同因子影响, 共同波动为 $e_{i, t}=0.7002 e_{i, t-1}+$ $0.0123 \varepsilon_{i, t}$.

但是注意到对深圳股市我们用 $r_{\alpha}=4$, 比较模拟结果, 可以认为深圳股市残差的方差大 于共同因子与因子负荷乘积的方差, 即可理解为深圳上市的股票相互影响不如股票本身前后 时刻的影响, 即深圳上市的股票主要靠本身的发展, 股票之间相互影响并不大, 产生这个结果 的另一个解释是因为股票数量不多的原因. 相反上海股市我们用 $r_{\alpha}=8$, 即股票间相互影响 大于或接近股票本身前后时刻的影响. 比较两个共同波动, 发现上海的共同波动性更大些, 即 可以理解为上海比深圳股市活跃.

\section{6 结束语}

因为大维因子分析比较复杂, 我们的模型只考虑了残差不相互独立而是服从一个具体的 线性时间序列模型的情形, 而且我们的结果并不完善, 例如没有给出任一 $\operatorname{ARMA}(p, q)$ 的参数 估计, 也没有给出模型的检验方面的理论, 这些都希望以后能有进一步的解决. 而且对很好的 模拟金融数据也有一定的差距, 因为金融数据是异方差, 具有短期惯性和长期反转等性质. 但 我们的结果考虑了金融市场上不同资产之间的相互影响和资产在不同时期的相互影响两个 重要方面, 这两个方面都与实际相符, 正如上面的模拟和实证我们能给出一些很好的理论结 果和对金融数据的解释. 


\section{参考文 献}

1 Bai J, Ng S. Determining the number of factors in approximate factor models. Econometrica, 70: 191-221 (2002)

2 Kapetanios G. A new method for determining the number of factors in factor models with large diastases. Working Paper. http : //www.econ.qmul.ac.uk/papers/doc/wp525.pdf, 2004

3 Onatski A. Determining the number of factors from empirical distribution of eigenvalues. Discussion Paper. http : //digitalcommons.libraries.columbia.edu/econ $\mathrm{d}_{\mathrm{p}} / 5 / 2005$

4 Bai J. Inferential theory for factor models of large dimensions. Econometrica, 71: 135-173 (2003)

5 Yin Y Q, Krishanaiah P R. A limit theorem for the eigenvalues of product of two random matrices. J Multivariate Anal, 13: 489-507 (1983)

6 Yin Y Q, Bai Z D, Krishanaiah P R. On the limit of the largest eigenvalue of the large dimensional sample covariance matrix. Probab Theory Related Fields, 78: 509-521 (1988)

7 Bai Z D, Miao B Q, Jin B S. On Limit theorem for the eigenvalues of product of two random matrices. $J$ Multivariate Anal, 98: 76-101 (2007)

8 Jegadeesh N, Titman S. Returns to buying winners and selling losers: Implications for stock market efficiency. Journal of Finance, 48: 65-91 (1993)

9 Yin Y Q. Limit spectral distribution for a class of random matrices. J Multivaritate Anal, 23: 50-68 (1986)

\section{附录}

表 $1 \quad \mathrm{MA}(1), e_{i, t}=a_{1} * \varepsilon_{i, t}+a_{2} * \varepsilon_{i, t-1}$

\begin{tabular}{|c|c|c|c|c|c|c|c|c|c|c|c|c|}
\hline \multicolumn{7}{|c|}{ 初值 } & \multicolumn{3}{|c|}{ 文献 [3] } & \multicolumn{3}{|c|}{ 本文结果 } \\
\hline$N$ & $T$ & $a_{1}$ & $a_{2}$ & $r$ & $r_{\max }$ & $r_{\alpha}$ & $\hat{r}_{0}$ & $\hat{r}_{N-1 / 2}$ & $\hat{r}_{N^{-2 / 5}}$ & $\hat{r}$ & $\hat{a}_{1}$ & $\hat{a}_{2}$ \\
\hline 200 & 60 & 1 & 0.5 & 1 & 8 & 8 & 1.16 & 1.03 & 1.00 & 1.00 & 1.00 & 0.49 \\
\hline 1000 & 60 & 1 & 0.5 & 1 & 8 & 8 & 1.01 & 1.00 & 1.00 & 1.00 & 1.00 & 0.49 \\
\hline 1000 & 250 & 1 & 0.5 & 1 & 15 & 8 & 1.16 & 1.00 & 1.00 & 1.00 & 1.00 & 0.50 \\
\hline 150 & 500 & 1 & 0.5 & 1 & 12 & 8 & 1.21 & 1.00 & 1.00 & 1.00 & 0.98 & 0.52 \\
\hline 150 & 150 & 1 & 0.5 & 1 & 12 & 8 & 1.47 & 1.03 & 1.00 & 1.01 & 0.99 & 0.50 \\
\hline 100 & 100 & 1 & 0.5 & 1 & 10 & 8 & 1.45 & 1.05 & 1.01 & 1.00 & 0.99 & 0.51 \\
\hline 40 & 100 & 1 & 0.5 & 1 & 7 & 8 & 1.32 & 1.01 & 1.00 & 1.01 & 0.94 & 0.57 \\
\hline 200 & 60 & 1.5 & 0.5 & 3 & 8 & 8 & 3.01 & 3.00 & 3.00 & 3.00 & 1.49 & 0.47 \\
\hline 1000 & 60 & 1.5 & 0.5 & 3 & 8 & 8 & 3.00 & 3.00 & 3.00 & 3.00 & 1.50 & 0.48 \\
\hline 1000 & 250 & 1.5 & 0.5 & 3 & 15 & 8 & 3.01 & 3.00 & 3.00 & 3.00 & 1.50 & 0.49 \\
\hline 150 & 500 & 1.5 & 0.5 & 3 & 12 & 8 & 3.02 & 3.00 & 3.00 & 3.00 & 1.45 & 0.58 \\
\hline 150 & 150 & 1.5 & 0.5 & 3 & 12 & 8 & 3.05 & 3.00 & 3.00 & 3.00 & 1.48 & 0.50 \\
\hline 100 & 100 & 1.5 & 0.5 & 3 & 10 & 8 & 3.05 & 3.00 & 3.00 & 3.00 & 1.47 & 0.50 \\
\hline 40 & 100 & 1.5 & 0.5 & 3 & 7 & 8 & 3.02 & 3.00 & 3.00 & 3.01 & 1.35 & 0.69 \\
\hline 200 & 60 & 2 & 0.5 & 5 & 8 & 8 & 5.00 & 5.00 & 5.00 & 5.00 & 1.98 & 0.43 \\
\hline 1000 & 60 & 2 & 0.5 & 5 & 8 & 8 & 5.00 & 5.00 & 5.00 & 5.00 & 2.00 & 0.46 \\
\hline 1000 & 250 & 2 & 0.5 & 5 & 15 & 8 & 5.00 & 5.00 & 5.00 & 5.00 & 2.00 & 0.49 \\
\hline 150 & 500 & 2 & 0.5 & 5 & 12 & 8 & 5.00 & 5.00 & 5.00 & 5.00 & 1.91 & 0.68 \\
\hline 150 & 150 & 2 & 0.5 & 5 & 12 & 8 & 5.00 & 5.00 & 5.00 & 5.00 & 1.96 & 0.50 \\
\hline 100 & 100 & 2 & 0.5 & 5 & 10 & 8 & 5.00 & 5.00 & 5.00 & 5.00 & 1.94 & 0.49 \\
\hline 40 & 100 & 2 & 0.5 & 5 & 7 & 8 & 5.00 & 5.00 & 5.00 & 5.00 & 1.68 & 0.92 \\
\hline
\end{tabular}


表 $2 \mathrm{MA}(1), e_{i, t}=a_{1} * \varepsilon_{i, t}+a_{2} * \varepsilon_{i, t-1}$

\begin{tabular}{|c|c|c|c|c|c|c|c|c|c|c|c|c|}
\hline \multicolumn{7}{|c|}{ 初值 } & \multicolumn{3}{|c|}{ 文献 [3] } & \multicolumn{3}{|c|}{ 本文结果 } \\
\hline$N$ & $T$ & $a_{1}$ & $a_{2}$ & $r$ & $r_{\max }$ & $r_{\alpha}$ & $\hat{r}_{0}$ & $\hat{r}_{N^{-1 / 2}}$ & $\hat{r}_{N}-2 / 5$ & $\hat{r}$ & $\hat{a}_{1}$ & $\hat{a}_{2}$ \\
\hline 200 & 60 & 3 & 0.5 & 1 & 8 & 6 & 1.17 & 1.02 & 1.00 & 1.06 & 2.99 & 0.50 \\
\hline 1000 & 60 & 3 & 0.5 & 1 & 8 & 8 & 1.043 & 1.01 & 1.00 & 1.00 & 3.00 & 0.49 \\
\hline 1000 & 250 & 3 & 0.5 & 1 & 15 & 8 & 1.15 & 1.01 & 1.00 & 1.00 & 3.00 & 0.50 \\
\hline 150 & 500 & 3 & 0.5 & 1 & 12 & 8 & 1.15 & 1.00 & 1.00 & 1.00 & 2.97 & 0.60 \\
\hline 150 & 150 & 3 & 0.5 & 1 & 12 & 8 & 1.34 & 1.01 & 1.00 & 1.01 & 2.98 & 0.52 \\
\hline 100 & 100 & 3 & 0.5 & 1 & 10 & 8 & 1.35 & 1.03 & 1.00 & 1.00 & 2.98 & 0.53 \\
\hline 40 & 100 & 3 & 0.5 & 1 & 7 & 8 & 1.20 & 1.00 & 1.00 & 1.00 & 2.90 & $0.71+0.01 \mathrm{i}$ \\
\hline 200 & 60 & 4.5 & 0.5 & 3 & 8 & 6 & 3.00 & 2.98 & 2.95 & 3.04 & 4.46 & $0.37+0.01 \mathrm{i}$ \\
\hline 1000 & 60 & 4.5 & 0.5 & 3 & 8 & 8 & 3.00 & 3.00 & 3.00 & 3.00 & 4.49 & 0.46 \\
\hline 1000 & 250 & 4.5 & 0.5 & 3 & 15 & 8 & 3.01 & 3.00 & 3.00 & 3.00 & 4.49 & 0.48 \\
\hline 150 & 500 & 4.5 & 0.5 & 3 & 12 & 8 & 3.01 & 3.00 & 3.00 & 3.00 & 4.39 & 0.90 \\
\hline 150 & 150 & 4.5 & 0.5 & 3 & 12 & 8 & 3.04 & 3.00 & 3.00 & 3.00 & 4.44 & 0.53 \\
\hline 100 & 100 & 4.5 & 0.5 & 3 & 10 & 6 & 3.03 & 2.99 & 2.94 & 3.00 & 4.41 & $0.50+0.02 \mathrm{i}$ \\
\hline 40 & 100 & 4.5 & 0.5 & 3 & 7 & 4 & 2.3 .0 & 1.59 & 1.31 & 4.17 & 4.12 & 1.22 \\
\hline 200 & 60 & 6 & 0.5 & 5 & 8 & 4 & 3.62 & 3.03 & 2.67 & 5.09 & 5.90 & $0.10+0.27 \mathrm{i}$ \\
\hline 1000 & 60 & 6 & 0.5 & 5 & 8 & 8 & 4.99 & 4.96 & 4.92 & 4.95 & 5.98 & 0.38 \\
\hline 1000 & 250 & 6 & 0.5 & 5 & 15 & 8 & 5.00 & 5.00 & 5.00 & 5.00 & 5.99 & 0.43 \\
\hline 150 & 500 & 6 & 0.5 & 5 & 12 & 8 & 5.00 & 5.00 & 5.00 & 5.00 & 5.75 & 1.36 \\
\hline 150 & 150 & 6 & 0.5 & 5 & 12 & 5 & 4.92 & 4.62 & 4.32 & 5.05 & 5.87 & $0.49+0.04 \mathrm{i}$ \\
\hline 100 & 100 & 6 & 0.5 & 5 & 10 & 4 & 3.48 & 2.63 & 2.15 & 4.66 & 5.78 & $0.39+0.13 \mathrm{i}$ \\
\hline 40 & 100 & 6 & 0.5 & 5 & 7 & 4 & 2.08 & 1.05 & 0.72 & 3.93 & 5.16 & 1.87 \\
\hline
\end{tabular}

表 $3 \quad \operatorname{AR}(1), e_{i, t}=b * e_{i, t-1}+a * \varepsilon_{i, t}$

\begin{tabular}{|c|c|c|c|c|c|c|c|c|c|c|c|c|}
\hline \multicolumn{7}{|c|}{ 初值 } & \multicolumn{3}{|c|}{ 文献 [3] } & \multicolumn{3}{|c|}{ 本文结果 } \\
\hline$N$ & $T$ & $a$ & $b$ & $r$ & $r_{\max }$ & $r_{\alpha}$ & $\hat{r}_{0}$ & $\hat{r}_{N-1 / 2}$ & $\hat{r}_{N-2 / 5}$ & $\hat{r}$ & $\hat{a}$ & $\hat{b}$ \\
\hline 200 & 60 & 1 & 0.5 & 1 & 8 & 8 & 1.326 & 1.099 & 1.029 & 1.007 & 0.99836 & 0.49378 \\
\hline 1000 & 60 & 1 & 0.5 & 1 & 8 & 8 & 1.001 & 1 & 1.001 & 1 & 1.001 & 0.49335 \\
\hline 1000 & 250 & 1 & 0.5 & 1 & 15 & 8 & 1.061 & 1.004 & 1 & 1 & 0.99969 & 0.49878 \\
\hline 150 & 500 & 1 & 0.5 & 1 & 12 & 8 & 1.392 & 1.006 & 1.001 & 1.006 & 0.99197 & 0.50637 \\
\hline 150 & 150 & 1 & 0.5 & 1 & 12 & 8 & 1.909 & 1.191 & 1.063 & 1.003 & 0.99548 & 0.49972 \\
\hline 100 & 100 & 1 & 0.5 & 1 & 10 & 8 & 2.036 & 1.328 & 1.099 & 1.002 & 0.99302 & 0.49959 \\
\hline 40 & 100 & 1 & 0.5 & 1 & 7 & 8 & 1.599 & 1.087 & 1.022 & 1.014 & 0.97444 & 0.51587 \\
\hline 200 & 60 & 1.5 & 0.5 & 3 & 8 & 8 & 3.016 & 3.001 & 3 & 3.003 & 1.4964 & 0.48531 \\
\hline 1000 & 60 & 1.5 & 0.5 & 3 & 8 & 8 & 3 & 3 & 3 & 3 & 1.5056 & 0.48577 \\
\hline 1000 & 250 & 1.5 & 0.5 & 3 & 15 & 8 & 3.006 & 3 & 3 & 3.001 & 1.5 & 0.49656 \\
\hline 150 & 500 & 1.5 & 0.5 & 3 & 12 & 8 & 3.039 & 3 & 3 & 3.003 & 1.47 & 0.51418 \\
\hline 150 & 150 & 1.5 & 0.5 & 3 & 12 & 8 & 3.203 & 3.013 & 3 & 3.002 & 1.4854 & 0.49725 \\
\hline 100 & 100 & 1.5 & 0.5 & 3 & 10 & 8 & 3.21 & 3.025 & 3.006 & 3.006 & 1.4768 & 0.49515 \\
\hline 40 & 100 & 1.5 & 0.5 & 3 & 7 & 8 & 3.071 & 3.003 & 3.001 & 3.005 & 1.4044 & 0.53144 \\
\hline 200 & 60 & 2 & 0.5 & 5 & 8 & 8 & 4.994 & 4.984 & 4.966 & 4.989 & 1.9913 & 0.47417 \\
\hline 1000 & 60 & 2 & 0.5 & 5 & 8 & 8 & 5 & 5 & 4.999 & 4.999 & 2.011 & 0.47776 \\
\hline 1000 & 250 & 2 & 0.5 & 5 & 15 & 8 & 5 & 5 & 5 & 5.002 & 2.0002 & 0.49457 \\
\hline 150 & 500 & 2 & 0.5 & 5 & 12 & 8 & 5.006 & 5 & 5 & 5.005 & 1.9349 & 0.52223 \\
\hline 150 & 150 & 2 & 0.5 & 5 & 12 & 8 & 5.013 & 5 & 5 & 5.002 & 1.969 & 0.49419 \\
\hline 100 & 100 & 2 & 0.5 & 5 & 10 & 8 & 5.009 & 5 & 5 & 5.012 & 1.9518 & 0.48992 \\
\hline 40 & 100 & 2 & 0.5 & 5 & 7 & 7 & 4.908 & 4.657 & 4.479 & 4.806 & 1.792 & 0.5463 \\
\hline
\end{tabular}


表 $4 \quad \operatorname{AR}(1), e_{i, t}=b * e_{i, t-1}+a * \varepsilon_{i, t}$

\begin{tabular}{ccccccc|ccc|ccc}
\hline \multicolumn{1}{c}{ 初值 } & \multicolumn{1}{c|}{ 文献 $[3]$} & \multicolumn{3}{c|}{ 本文结果 } \\
\hline$N$ & $T$ & $a$ & $b$ & $r$ & $r_{\max }$ & $r_{\alpha}$ & $\hat{r}_{0}$ & $\hat{r}_{N-1 / 2}$ & $\hat{r}_{N-2 / 5}$ & $\hat{r}$ & $\hat{a}$ & $\hat{b}$ \\
\hline 200 & 60 & 3 & 0.5 & 1 & 8 & 6 & 1.3 & 1.087 & 1.019 & 1.043 & 2.9904 & 0.4933 \\
1000 & 60 & 3 & 0.5 & 1 & 8 & 8 & 1 & 1 & 0.999 & 0.996 & 3.0005 & 0.4928 \\
1000 & 250 & 3 & 0.5 & 1 & 15 & 8 & 1.05 & 1.006 & 1 & 1 & 2.9989 & 0.49871 \\
150 & 500 & 3 & 0.5 & 1 & 12 & 8 & 1.378 & 1.005 & 1 & 1.002 & 2.9755 & 0.50622 \\
150 & 150 & 3 & 0.5 & 1 & 12 & 8 & 1.924 & 1.223 & 1.048 & 1.001 & 2.9849 & 0.49959 \\
100 & 100 & 3 & 0.5 & 1 & 10 & 6 & 2.035 & 1.304 & 1.123 & 1.099 & 2.9768 & 0.4976 \\
40 & 100 & 3 & 0.5 & 1 & 7 & 6 & 1.604 & 1.084 & 1.019 & 1.024 & 2.9227 & 0.5105 \\
200 & 60 & 4.5 & 0.5 & 3 & 8 & 4 & 1.463 & 0.955 & 0.719 & 3.358 & 4.4523 & 0.4674 \\
1000 & 60 & 4.5 & 0.5 & 3 & 8 & 4 & 0.936 & 0.725 & 0.578 & 2.937 & 4.4797 & 0.4782 \\
1000 & 250 & 4.5 & 0.5 & 3 & 15 & 8 & 3.005 & 3.001 & 3 & 3.002 & 4.4975 & 0.49632 \\
150 & 500 & 4.5 & 0.5 & 3 & 12 & 8 & 3.055 & 3 & 3 & 3.003 & 4.4073 & 0.51362 \\
150 & 150 & 4.5 & 0.5 & 3 & 12 & 5 & 3.12 & 2.836 & 2.635 & 2.583 & 4.4454 & 0.4927 \\
100 & 100 & 4.5 & 0.5 & 3 & 10 & 5 & 2.615 & 1.875 & 1.597 & 4.039 & 4.4159 & 0.4823 \\
40 & 100 & 4.5 & 0.5 & 3 & 7 & 4 & 1.595 & 0.656 & 0.398 & 3.613 & 4.2092 & 0.4925 \\
200 & 60 & 6 & 0.5 & 5 & 8 & 4 & 0.911 & 0.44 & 0.22 & 3.236 & 5.7985 & 0.4251 \\
1000 & 60 & 6 & 0.5 & 5 & 8 & 4 & 0.18 & 0.09 & 0.04 & 2.342 & 5.8833 & 0.4505 \\
1000 & 250 & 6 & 0.5 & 5 & 15 & 8 & 5 & 4.994 & 4.984 & 4.989 & 5.9914 & 0.49305 \\
150 & 500 & 6 & 0.5 & 5 & 12 & 6 & 4.996 & 4.904 & 4.659 & 4.911 & 5.7986 & 0.5196 \\
150 & 150 & 6 & 0.5 & 5 & 12 & 8 & 2.997 & 2.148 & 1.667 & 3.745 & 5.8832 & 0.4772 \\
100 & 100 & 6 & 0.5 & 5 & 10 & 8 & 2.72 & 1.59 & 1.093 & 3.281 & 5.7896 & 0.4469 \\
40 & 100 & 6 & 0.5 & 5 & 7 & 4 & 1.246 & 0.353 & 0.171 & 3.407 & 5.2929 & 0.47723 \\
\hline
\end{tabular}

表 $5 \quad \hat{r}_{1}=\hat{r}_{N^{-1 / 2}}, \hat{r}_{2}=\hat{r}_{N^{-1 / 2}}, e_{i, t}=b_{1} e_{i, t-1}+a_{1} \varepsilon_{i, t}+a_{2} \varepsilon_{i, t-1}$

\begin{tabular}{cccccccc|cccc|ccc}
\hline \multicolumn{1}{c|}{ 初值 } & \multicolumn{1}{c|}{ 文献 $[3]$} & \multicolumn{3}{c|}{ 本文结果 } \\
\hline$N$ & $T$ & $a_{1}$ & $b_{1}$ & $a_{2}$ & $r$ & $r_{\max }$ & $r_{\alpha}$ & $\hat{r}_{0}$ & $\hat{r}_{1}$ & $\hat{r}_{2}$ & $\hat{r}$ & $\hat{a}_{1}$ & $\hat{b}_{1}$ & $\hat{a}_{2}$ \\
\hline 200 & 60 & 1 & 0.1 & 0.5 & 1 & 8 & 8 & 1.3 & 1 & 1 & 1 & 0.97 & 0.20 & 0.41 \\
1000 & 60 & 1 & 0.1 & 0.5 & 1 & 8 & 8 & 1.2 & 1 & 1 & 1 & 1.1 & 0.94 & -0.91 \\
1000 & 250 & 1 & 0.1 & 0.5 & 1 & 15 & 8 & 1.4 & 1 & 1 & 1 & 0.97 & 0.09 & 0.55 \\
150 & 500 & 1 & 0.1 & 0.5 & 1 & 12 & 8 & 1.12 & 1 & 1 & 1 & 1.00 & 0.11 & 0.48 \\
150 & 150 & 1 & 0.1 & 0.5 & 1 & 12 & 8 & 1.41 & 1.1 & 1 & 1.0 & 1.00 & 0.12 & 0.47 \\
100 & 100 & 1 & 0.1 & 0.5 & 1 & 10 & 8 & 1.4 & 1 & 1 & 1 & 1.00 & 0.13 & 0.46 \\
40 & 100 & 1 & 0.1 & 0.5 & 1 & 7 & 8 & 1.3 & 1.1 & 1 & 1 & 1.01 & 0.14 & 0.42 \\
200 & 60 & 1.5 & 0.5 & 0.1 & 3 & 8 & 8 & 3.1 & 3 & 3 & 3.01 & 1.28 & 0.39 & 0.57 \\
1000 & 60 & 1.5 & 0.5 & 0.1 & 3 & 8 & 8 & 3 & 3 & 3 & 3 & 1.53 & 0.91 & -1.03 \\
1000 & 250 & 1.5 & 0.5 & 0.1 & 3 & 15 & 8 & 3.2 & 3 & 3 & 3 & 1.46 & 0.48 & 0.19 \\
150 & 500 & 1.5 & 0.5 & 0.1 & 3 & 12 & 8 & 3 & 3 & 3 & 3 & 1.50 & 0.50 & 0.08 \\
150 & 150 & 1.5 & 0.5 & 0.1 & 3 & 12 & 8 & 3.2 & 3 & 3 & 3 & 1.49 & 0.51 & 0.07 \\
100 & 100 & 1.5 & 0.5 & 0.1 & 3 & 10 & 8 & 3.2 & 3 & 3 & 3 & 1.48 & 0.51 & 0.05 \\
40 & 100 & 1.5 & 0.5 & 0.1 & 3 & 7 & 8 & 3.1 & 2.9 & 2.9 & 2.9 & 1.49 & 0.48 & 0.07 \\
200 & 60 & 2 & 0.3 & 0.5 & 5 & 8 & 8 & 5 & 5 & 5 & 5.01 & $1.60+0.25 \mathrm{i}$ & 0.15 & $1.29-0.25 \mathrm{i}$ \\
1000 & 60 & 2 & 0.3 & 0.5 & 5 & 8 & 8 & 5 & 5 & 5 & 5 & 1.99 & 0.96 & -1.64 \\
1000 & 250 & 2 & 0.3 & 0.5 & 5 & 15 & 8 & 5 & 5 & 5 & 5 & $1.67+0.03 \mathrm{i}$ & 0.12 & $1.33-0.03 \mathrm{i}$ \\
150 & 500 & 2 & 0.3 & 0.5 & 5 & 12 & 8 & 5 & 5 & 5 & 5 & 2.00 & 0.30 & 0.46 \\
150 & 150 & 2 & 0.3 & 0.5 & 5 & 12 & 8 & 5 & 5 & 5 & 5 & 1.97 & 0.32 & 0.43 \\
100 & 100 & 2 & 0.3 & 0.5 & 5 & 10 & 8 & 5 & 5 & 5 & 5 & 1.96 & 0.30 & 0.46 \\
40 & 100 & 2 & 0.3 & 0.5 & 5 & 7 & 7 & 4.4 & 4.1 & 3.9 & 4.46 & 1.97 & 0.26 & 0.45 \\
\hline
\end{tabular}


表 $6 \quad \hat{r}_{1}=\hat{r}_{N^{-1 / 2}}, \hat{r}_{2}=\hat{r}_{N^{-1 / 2}}, e_{i, t}=b_{1} e_{i, t-1}+a_{1} \varepsilon_{i, t}+a_{2} \varepsilon_{i, t-1}$

\begin{tabular}{|c|c|c|c|c|c|c|c|c|c|c|c|c|c|c|}
\hline \multicolumn{8}{|c|}{ 初值 } & \multicolumn{3}{|c|}{ 文献 [3] } & \multicolumn{4}{|c|}{ 本文结果 } \\
\hline$N$ & $T$ & $a_{1}$ & $b_{1}$ & $a_{2}$ & $r$ & $r_{\max }$ & $r_{\alpha}$ & $\hat{r}_{0}$ & $\hat{r}_{1}$ & $\hat{r}_{2}$ & $\hat{r}$ & $\hat{a}_{1}$ & $\hat{b}_{1}$ & $\hat{a}_{2}$ \\
\hline 200 & 60 & 3 & 0.1 & 0.5 & 1 & 8 & 6 & 1.2 & 1 & 1 & 1.02 & 2.96 & 0.49 & -0.76 \\
\hline 1000 & 60 & 3 & 0.1 & 0.5 & 1 & 8 & 8 & 1 & 1 & 1 & 1 & 3.02 & 0.99 & -2.91 \\
\hline 1000 & 250 & 3 & 0.1 & 0.5 & 1 & 15 & 8 & 1.2 & 1 & 1 & 1 & 2.98 & 0.19 & 0.28 \\
\hline 150 & 500 & 3 & 0.1 & 0.5 & 1 & 12 & 8 & 1.1 & 1 & 1 & 1 & 3.00 & 0.11 & 0.46 \\
\hline 150 & 150 & 3 & 0.1 & 0.5 & 1 & 12 & 8 & 1.4 & 1 & 1 & 1 & 2.99 & 0.20 & 0.16 \\
\hline 100 & 100 & 3 & 0.1 & 0.5 & 1 & 10 & 8 & 1.5 & 1.1 & 1 & 1 & 2.99 & 0.29 & -0.15 \\
\hline 40 & 100 & 3 & 0.1 & 0.5 & 1 & 7 & 8 & 1.3 & 1 & 1 & 1 & 2.98 & 0.23 & 0.03 \\
\hline 200 & 60 & 4.5 & 0.5 & 0.1 & 3 & 8 & 4 & 2.1 & 1.3 & 1 & 2.6 & $3.70+0.3 \mathrm{i}$ & 0.26 & $2.33-0.3 \mathrm{i}$ \\
\hline 1000 & 60 & 4.5 & 0.5 & 0.1 & 3 & 8 & 8 & 3 & 3 & 2.8 & 3 & 4.58 & 0.93 & -3.42 \\
\hline 1000 & 250 & 4.5 & 0.5 & 0.1 & 3 & 15 & 8 & 3 & 3 & 3 & 3 & 4.33 & 0.42 & 0.81 \\
\hline 150 & 500 & 4.5 & 0.5 & 0.1 & 3 & 12 & 8 & 3 & 3 & 3 & 2.9 & 4.49 & 0.50 & 0.04 \\
\hline 150 & 150 & 4.5 & 0.5 & 0.1 & 3 & 12 & 5 & 2.9 & 2.1 & 1.7 & 2.64 & 4.45 & 0.49 & 0.10 \\
\hline 100 & 100 & 4.5 & 0.5 & 0.1 & 3 & 10 & 5 & 2.3 & 1.5 & 1.2 & 1.7 & 4.38 & 0.44 & 0.36 \\
\hline 40 & 100 & 4.5 & 0.5 & 0.1 & 3 & 7 & 4 & 1.5 & 0.9 & 0.7 & 3.8 & 4.34 & 0.40 & 0.31 \\
\hline 200 & 60 & 6 & 0.3 & 0.5 & 5 & 8 & 4 & 2.9 & 1.9 & 1.5 & 4.7 & 5.56 & 0.57 & -1.16 \\
\hline 1000 & 60 & 6 & 0.3 & 0.5 & 5 & 8 & 6 & 5 & 5 & 4.6 & 5.4 & 5.7 & 0.97 & -4.97 \\
\hline 1000 & 250 & 6 & 0.3 & 0.5 & 5 & 15 & 6 & 5 & 5 & 5 & 5 & 5.66 & 0.10 & 2.37 \\
\hline 150 & 500 & 6 & 0.3 & 0.5 & 5 & 12 & 6 & 5 & 5 & 4.8 & 5 & 5.98 & 0.30 & 0.39 \\
\hline 150 & 150 & 6 & 0.3 & 0.5 & 5 & 12 & 5 & 3.7 & 3 & 2.5 & 3.3 & 5.86 & 0.26 & 0.70 \\
\hline 100 & 100 & 6 & 0.3 & 0.5 & 5 & 10 & 4 & 2.5 & 1.6 & 1.2 & 4 & 5.76 & 0.17 & 1.13 \\
\hline 40 & 100 & 6 & 0.3 & 0.5 & 5 & 7 & 4 & 1.2 & 0.6 & 0.4 & 3.35 & 5.71 & 0.18 & 0.52 \\
\hline
\end{tabular}

